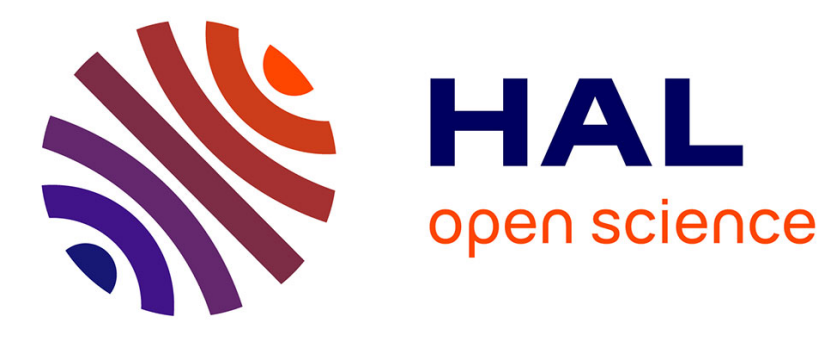

\title{
Stability of the polydomain state in epitaxial ferroelectric $\mathrm{PbTiO} 3$ films
}

\author{
Ausrine Bartasyte, B. Dkhil, Jens Kreisel, J. Chevreul, Odette \\ Chaix-Pluchery, L. Rapenne-Homand, Carmen Jiménez, Adulfas Abrutis, \\ François Weiss
}

\section{To cite this version:}

Ausrine Bartasyte, B. Dkhil, Jens Kreisel, J. Chevreul, Odette Chaix-Pluchery, et al.. Stability of the polydomain state in epitaxial ferroelectric PbTiO3 films. Applied Physics Letters, 2008, 93, pp.242907. 10.1063/1.3046787 . hal-00411750

\section{HAL Id: hal-00411750 https://hal.science/hal-00411750}

Submitted on 28 Aug 2009

HAL is a multi-disciplinary open access archive for the deposit and dissemination of scientific research documents, whether they are published or not. The documents may come from teaching and research institutions in France or abroad, or from public or private research centers.
L'archive ouverte pluridisciplinaire HAL, est destinée au dépôt et à la diffusion de documents scientifiques de niveau recherche, publiés ou non, émanant des établissements d'enseignement et de recherche français ou étrangers, des laboratoires publics ou privés. 


\title{
Stability of the polydomain state in epitaxial ferroelectric $\mathrm{PbTiO}_{3}$ films
}

\author{
A. Bartasyte ${ }^{1,2}$ a) , B. Dkhil ${ }^{3}$, J. Kreisel ${ }^{1}$, J. Chevreul ${ }^{3}$, O. Chaix-Pluchery ${ }^{1}$, L. Rapenne- \\ Homand $^{1}$, C. Jimenez ${ }^{1}$, A. Abrutis ${ }^{2}$, and F. Weiss ${ }^{1}$ \\ ${ }^{1}$ Laboratoire des Matériaux et du Génie Physique (CNRS UMR 5628), Minatec, Grenoble \\ Institute of Technology, 3 parvis Louis Néel, 38016 Grenoble, France \\ ${ }^{2}$ Vilnius University, Dept. of General and Inorganic Chemistry, LT-03225 Vilnius, Lithuania \\ ${ }^{3}$ Laboratoire Structures, Propriétés et Modélisation des Solides (CNRS-Ecole Centrale \\ Paris), 92295 Châtenay-Malabry Cedex, France \\ a) Electronic address: a.bartasyte1@physics.ox.ac.uk
}

\begin{abstract}
Phase transitions and domain state transformations in a $250 \mathrm{~nm}$ thick $\mathrm{PbTiO}_{3} / \mathrm{SrTiO}_{3}$ film with $c / a / c / a$ domain structure were investigated by temperature-dependent X-ray diffraction and Raman spectroscopy. Both techniques provide evidence that the film remains tetragonal up to 650 ${ }^{\circ} \mathrm{C}$ while the bulk sample becomes cubic at $490{ }^{\circ} \mathrm{C}$. Small anomalies in the width of both Bragg peaks and in Raman modes suggest a subtle structural rearrangement around $500{ }^{\circ} \mathrm{C}$.
\end{abstract}


Phase transitions in ferroelectric (FE) $\mathrm{PbTiO}_{3}$ (PTO) single crystals have been studied extensively by various techniques: X-ray diffraction (XRD) $)^{1,2}$, Raman spectroscopy ${ }^{3-4}$, extended X-ray absorption fine structure (EXAFS $)^{5}$, neutron diffraction ${ }^{6}$, birefringence $^{2}$ etc. and have led to a deeper understanding of ferroelectric instabilities, which have numerous applications. Phase transitions in ferroelectric thin films and, in particular, epitaxial thin films have been studied much less. Epitaxial films are bound to the underlying substrate; therefore, the in-plane lattice parameters of the films are strained, which prevents them from adopting the normal bulk values. This is known as the substrate clamping effect, which forces the in-plane lattice parameters of films to follow the temperature behaviour of the substrate. It has already been reported that this has an important effect on phase transitions and domain formation in thin films ${ }^{7-10}$. The out-ofplane lattice parameter of the film is thought to compensate the in-plane strain in order to accommodate the volume change necessary for the phase transition by means of stresses which can modify the phase transition order, the transition temperature and phase sequence, or decouple the primary and the secondary order parameters ${ }^{7,10,11}$.

In the recent years theoretical efforts have been made to understand the effects of strain on the phase transitions in epitaxial FE films, such as PTO or $\mathrm{PbZr}_{1-\mathrm{x}} \mathrm{Ti}_{\mathrm{x}} \mathrm{O}_{3}(\mathrm{PZT})$. By using the LandauGinzburg-Devonshire type nonlinear phenomenological theory, Pertsev and coworkers ${ }^{12}$ developed the concept of a misfit strain-temperature diagram for FE polydomain and singledomain PTO films. According to this theoretical framework, the FE-paraelectric (FE-PE) phase transition temperature increases when films are subjected to tensile or compressive stresses, in agreement with experimental results obtained for strained single-domain $\mathrm{PTO} / \mathrm{PZT}^{9,10,13}$ or other perovskite-type oxide epitaxial films $s^{7,8,11,14}$.

However, only limited data are available on domain state transformations and phase transitions in partially relaxed and polydomain films. In this study, the influence of the substrate on the domain state transformations and FE-PE phase transition of partially relaxed epitaxial $\mathrm{PTO} / \mathrm{SrTiO}_{3}$ (STO) films, with the $c / a / c / a$ polydomain state, was investigated from room temperature (RT) to $650^{\circ} \mathrm{C}$ by using XRD and Raman spectroscopy.

A $250 \mathrm{~nm}$ thick PTO film was deposited at $650{ }^{\circ} \mathrm{C}$ on (00l) oriented STO single-crystal substrates by pulsed injection metalorganic chemical vapour deposition ${ }^{15}$. The full width at half maximum (FWHM) of the film (001) reflection rocking curve corresponds to the instrumental resolution of the diffractometer $\left(0.1^{\circ}\right)$, thus confirming the excellent epitaxial quality of the film. At RT, the $a$-domain fraction is very small $(\sim 4 \pm 1 \%)$ in this film and has been estimated from the ratio of (102) reflection intensities of $a$ - and $c$-domains in $\varphi$-scans ${ }^{15}$. 
High-temperature XRD measurements in the temperature range from RT to $650{ }^{\circ} \mathrm{C}$ were performed using a high-resolution goniometer $\left(1 \mathrm{step}=0.0002^{\circ}\right.$ in $\left.2 \Theta\right)$ with an $18 \mathrm{~kW}$ rotating $\mathrm{Cu}$ anode generator $\left(\lambda_{C u K_{\beta}}=1.39223 \AA\right)$. The maximum temperature of our investigation was limited to $650^{\circ} \mathrm{C}$, i.e., the growth temperature in order to avoid any transformation (deterioration, appearance of defects, domain state rearrangement, etc.) within the film. The $c$ - and $a$-lattice parameters were calculated from the (004) and (400) reflections in $\Theta / 2 \Theta$ standard scans of $c$ - and $a$-domains, respectively. It was assumed ${ }^{16,17}$ that the out-of-plane $a$-parameter of $a$-domains is the same as the in-plane $a$-parameter of $c$-domains. The evolution of the fraction of $a$-domains with temperature was evaluated from the ratio of (400) and (004) reflection intensities.

Raman spectra, in crossed $(V H)$ and parallel $(V V)$ polarization configurations, were collected

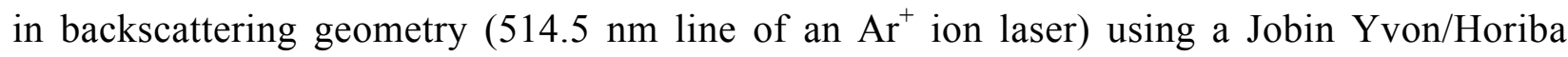
LabRam spectrometer equipped with a liquid nitrogen cooled charge coupled device detector. The temperature measurements in the range from RT to $600^{\circ} \mathrm{C}$ were carried out using a commercial LINKAM heating stage.

The quality of the film-substrate interface was studied by transmission electron microscopy (TEM) JEOL 2011. Samples of the cross section of the interface between the film and the substrate were prepared by mechanical polishing and finally, ion beam polishing.

The high-resolution cross-section TEM image of the PTO/STO film is shown in Figs. 1(a) and 1(b). The $c$-domain interface is coherent without any misfit dislocation in this region, confirming a high epitaxial quality of the film. Electron diffraction patterns taken at the interface (Fig. 1(c)), confirmed the in-plane coherence, without any splitting of the (0k0) PTO and STO reflections up to the third order. Such high quality interfaces are similar to those reported in the literature for very thin epitaxial films ${ }^{18}$. No splitting of (h00) PTO and STO was observed by XRD when probing all the film thicknesses up to $250 \mathrm{~nm}$ thick, not shown here, indicating a high quality of the whole film and not only of the interface.

The formation of $a$-domains is less likely in film on an STO substrate due to a relatively important lattice mismatch (- $6.3 \%$ at RT). As seen in Fig. 1(a), the interface in the $250 \mathrm{~nm}$ thick film between the $a$-domains and the substrate presents defects. As a consequence of this, a part of the compressive misfit stress is expected to be already relaxed by creation of dislocations at the interface. This, in turn, provides nucleation sites for a-domains during cooling through the FE-PE phase transition after the sample synthesis. The average domain period $W$, estimated from lower magnification TEM images, is about $240 \mathrm{~nm}$, which corresponds roughly to the thickness of our film. 
The change in $a$ - and $c$ - lattice parameters of the PTO film with temperature, as determined by $\mathrm{XRD}$, is shown in Fig. 2(a) in comparison with the values for a bulk PTO powder, taken from Ref. 2. In agreement with the literature ${ }^{2}$ we observe that the tetragonality of the powder decreases progressively with increasing temperature, and finally vanishes at about $T_{C \text {,bulk }}=490{ }^{\circ} \mathrm{C}$, which corresponds to the well-known bulk FE-tetragonal to PE-cubic phase transition. The temperature evolution of lattice parameters in the PTO film is similar to that in bulk samples in the sense that the tetragonality decreases with increasing temperature (Figs. 2(a) and (2b)). However in the case of the thin film the tetragonality does not vanish at $T_{C, b u l k}$ but persists up to the highest investigated temperature of $650{ }^{\circ} \mathrm{C}$, i.e. $160{ }^{\circ} \mathrm{C}$ higher than $T_{C, b u l k}$. By assuming either the same type of first-order phase transition as in the bulk or a more continuous second-order transition, the extrapolation of the tetragonality $(c / a-1)$ to zero indicates that a cubic-like phase transition is likely to occur at $T_{C, f i m} \approx 750{ }^{\circ} \mathrm{C}$ or at $T_{C, f i l m} \approx 900{ }^{\circ} \mathrm{C}$, respectively (Fig. 2(b)). XRD suggests that a polydomain structure is maintained at high temperature, and only the relative volume fraction between $c$ - and $a$-domains changes. However the FWHM of the (004) reflection in Fig. 2(c) shows an anomaly at $\sim 490{ }^{\circ} \mathrm{C}$, indicating the possibility of a subtle structural rearrangement at this temperature, which is surprisingly close to $T_{C, b u l k}$.

To support such a scenario, we also carried out a Raman study of our PTO film as a function of temperature. There is no report in literature of a Raman study of PTO (PZT) films on STO substrates. Unfortunately, in the conventional scattering geometry with the laser perpendicular to the film surface, the PTO Raman signature is masked by an important scattering from the STO substrate, which might explain the lack of Raman data in literature. One way to overcome this problem is to use an ultraviolet laser as was demonstrated by Tenne et $a l .{ }^{19}$ for $\mathrm{BaTiO}_{3}$ films on a STO substrate. We chose an alternative way of decreasing the STO signal by collecting polarized Raman spectra of the film cross-section (i.e., the laser beam parallel to the film surface, Fig. 3(c)). The Raman spectra, collected on the surface of $(100) /(001)$ PTO film, are dominated by $a$ domains (for more details see Ref. 20) while Raman measurements of the film cross section allow the observation of $c$-domain modes. Thus, this geometry is fundamental for the analysis of films with a dominant $c$-domain fraction, as in this case. Figure 3(a) shows the change with temperature of $V H$ polarized Raman spectra of the PTO film. The $E(T O)$ hard modes in $V H$ spectra remain well defined and intense up to $600^{\circ} \mathrm{C}$ except the $E(3 T O)$ mode (Fig. 3(a)). This observation provides further evidence that the tetragonal structure is maintained in the temperature range investigated, without any FE-PE phase transition for $250 \mathrm{~nm}$ thick PTO/STO films. The change with temperature of $E(T O)$ mode wavenumbers displays a slight but significant change of the slope around $500{ }^{\circ} \mathrm{C}$ (Fig. 3(b)). This anomaly is only subtle, but similar slight 
changes have also been observed in our XRD data, thus pointing at a structural rearrangement that needs further investigation.

The misfit strain $S_{m}$, as defined by Pertsev ${ }^{12}$,

$$
S_{m}=A\left(a_{S T O}-c_{f}\right) / a_{S T O}+(1-A)\left(a_{S T O}-a_{f}\right) / a_{S T O}
$$

where $a_{f}$ and $c_{f}$ are in-plane and out-of-plane lattice parameters of the film, $a_{S T O}$ the lattice parameter of the STO substrate, and $A$ the $a$-domain fraction, which changes from $-0.3 \%$ to -0.6 $\%$ when heating from 70 to $550{ }^{\circ} \mathrm{C}$ (Fig. 2(d)). At the same time, the $a$-domain fraction increases up to $17 \%$ at $550{ }^{\circ} \mathrm{C}$. Above this temperature, it becomes difficult to unambiguously determine the domain fraction as the (400) reflection from the film starts to overlap the reflection from the substrate. The $T_{c}$ obtained by extrapolation towards high temperatures of the misfit strain in the Pertsev phase diagram is close to $600{ }^{\circ} \mathrm{C}$ (Fig. 2(d)), i.e., a temperature lower than that obtained from the change in tetragonality with temperature (Fig. 2(b)). This discrepancy might arise from surface/interface effects ${ }^{21}$ which would shift the calculated phase diagram with respect to the zero misfit value and change the $T_{c}$ values. Indeed, shifts of $T_{c}$ to higher temperatures have been reported for single-domain PZT and PTO films ${ }^{9,10,13}$. However our film has a $c / a / c / a$ polydomain state in the whole temperature range investigated.

Interestingly, the Pertsev phase diagram predicts a $c / a / c / a$ domain up to $500{ }^{\circ} \mathrm{C}$, which is consistent with our results. However, at $\sim 500{ }^{\circ} \mathrm{C}$ (the temperature where our anomalies in XRD and Raman data are observed) $S_{m}(T)$ crosses from the $c / a / c / a$ to the $c$-domain stability boundary, while our films had a $c / a / c / a$ domain structure over the whole temperature range. Theory predicts the stability of $c$ - or $a_{1} / a_{2} / a_{1} / a_{2}$ phases at high temperatures and the stability of the $c / a / c / a$ domain structure only below the bulk PTO phase transition temperature $\left(490{ }^{\circ} \mathrm{C}\right)$. It is possible that our film exhibits the coexistence of the upper $c$ or $a_{1} / a_{2} / a_{1} / a_{2}$ phase with the lower temperature $c / a / c / a$ domain structure, as in a first-order phase transition even if Pertsev's phase diagram indicates a second-order type transition. Besides, our film thickness $H$ is in the order of magnitude of the domain period ( $W=240 \mathrm{~nm}$ ) leading to highly inhomogeneous internal polarization and strain fields. This might explain the deviation observed from the theoretical Pertsev's phase diagrams developed for dense domain structures, where $W<<H$. It was proved that the Pertsev diagrams give a good prediction of the stability of the domain structures in polydomain $\mathrm{PTO} / \mathrm{MgO}$ or $\mathrm{PZT} / \mathrm{MgO}$ films, where the a-domain fraction is bigger $(25-28 \%$ at $\mathrm{RT}$ ) and thus the domain period is much smaller than that in PTO/STO films. ${ }^{16,22}$

In conclusion, we have investigated phase transitions and domain state transformations in a $250 \mathrm{~nm}$ thick PTO/STO film with the $c / a / c / a$ domain structure. Both XRD and Raman spectroscopy results indicate that such films are tetragonal and contain both $a$ - and $c$-domains 
from RT to $650{ }^{\circ} \mathrm{C}$, whereas theory predicts the stability of the $c / a / c / a$ domain state only below the phase transition temperature in bulk PTO. Some structural rearrangement is likely to occur at about $500{ }^{\circ} \mathrm{C}$, indicating a possible transition to a tetragonal PE phase or some kind of domain state transformation. These observations are at variance with the Pertsev diagram for polydomain PTO films and indicate either the need for further experimental structural investigation into polydomain films or theoretical calculations, beyond the $W<<H$ approximation, by modeling the strain from first principles. ${ }^{23}$

Acknowledgments: This work has been financially supported by the European network of excellence FAME and EGIDE. 


\section{References}

${ }^{1}$ G. Shirane and S. Hoshino, J. Phys. Soc. Jpn. 6, 265 (1951).

2 S.A. Mabud and A.M. Glazer, J. Appl. Crystallogr. 12, 49 (1979).

${ }^{3}$ G. Burns and B.A. Scott, Phys. Rev. Lett. 25, 167 (1970).

${ }^{4}$ M.D. Fontana, H. Idrissi, G.E. Kugel, and K. Wojcik, Ferroelectrics 80, 117 (1988).

${ }^{5}$ Y. Yacoby, Y. Girshberg and E.A. Stern, Z. Phys. B: Condens. Matter 104, 725 (1997).

${ }^{6}$ R.J. Nelmes and W.F. Kuhs, Solid State Commun. 54, 721 (1985).

${ }^{7}$ F. He and B.O. Wells, Appl. Phys. Lett. 88, 152908 (2006).

${ }^{8}$ K.J. Choi, M. Biegalski, Y.L. Li, A. Sharan, J. Shubert, R. Uecker, P. Reiche, Y.B. Chen, X.Q.

Pan, V. Gopalan, L.-Q. Chen, D.G. Schlom, and C.B. Eom, Science 306, 1005 (2004).

${ }^{9}$ P.-E. Janolin, F. Le Marrec, J. Chevreul, and B. Dkhil, Appl. Phys. Lett. 90, 192910 (2007).

${ }^{10}$ P.-E. Janolin, B. Fraisse, F. Le Marrec, and B. Dkhil, Appl. Phys. Lett. 90, 212904 (2007).

${ }^{11}$ Yu. I. Yuzyuk, P. Simon, I.N. Zakharchenko, V.A. Alyoshin, and E.V. Sviridov, Phys. Rev. B 66, 052103 (2002).

${ }^{12}$ N. Pertsev, V. Khukar, H. Kohlstedt, and R. Waser. Phys. Rev. B 67, 054107 (2003), and references therein.

${ }^{13}$ P. Paruch and J.M. Triscone, Appl. Phys. Lett. 88, 162907 (2006).

${ }^{14}$ J.H. Haeni, P. Irvin, W. Chang, R. Uecker, P. Reiche, Y.L. Li, S. Choudhury, W. Tian, M.E. Hawley, B. Craigo, A. K. Tagantsev, X.Q. Pan, S.K. Streiffer, L.Q. Chen, S.W. Kirchoefer, J. Levy, and D.G. Schlom, Nature 430, 758 (2004).

${ }^{15}$ A. Bartasyte, A. Abrutis, C. Jimenez, F. Weiss, O Chaix-Pluchery, and Z. Saltyte, Ferroelectrics 353, 104 (2007).

${ }^{16}$ P.-E. Janolin, B. Fraisse, B. Dkhil, F. Le Marrec, and E. Ringgaard, Appl. Phys. Lett. 90, 162906 (2007).

${ }^{17}$ K.S. Lee, J.H. Choi, J.Y. Lee, and S. Baik, J. Appl. Phys. 90, 4095 (2001).

${ }^{18}$ A.T.J. van Helvoort, O. Dahl, B.G. Soleim, R. Holmestad, and T. Tybell, Appl. Phys. Lett. 86, 092907 (2005).

19 D. A. Tenne, A. Bruchhausen, N. D. Lanzillotti-Kimura, A. Fainstein, R. S. Katiyar, A. Cantarero, A. Soukiassian, V. Vaithyanathan, J. H. Haeni, W. Tian, D. G. Schlom, K. J. Choi, D. M. Kim, C. B. Eom, H. P. Sun, X. Q. Pan, Y. L. Li, L. Q. Chen, Q. X. Jia, S. M. Nakhmanson, K. M. Rabe, and X. X. Xi, Science 313, 1614 (2006). 
${ }^{20}$ A. Bartasyte, O. Chaix-Pluchery, J. Kreisel, J. Santiso, M. Boudard, C. Jimenez, A. Abrutis, and F. Weiss, IEEE Trans. Ultrason. Ferroelectr. Freq. Control 54, 2623 (2007).

${ }^{21}$ B.-K. Lai, J.A. Kornev, L. Bellaiche, and G.J. Salamo, Appl. Phys. Lett. 86, 132904 (2005).

${ }^{22}$ K. Lee and S. Baik, Annu. Rev. Mater. Res. 36, 81 (2006).

${ }^{23}$ O. Dieguez and D. Vanderbilt, Phase Transitions 81, 607 (2008). 


\section{Figure captions}

Figure 1 (a) High resolution cross-sectional TEM micrograph of the $250 \mathrm{~nm}$ thick PTO/STO film. (b) Magnified TEM micrograph and (c) electron diffraction pattern measured at the interface of the $c$-domain and the STO substrate.

Figure 2 (a) Temperature evolution of lattice parameters and (b) the tetragonality in a $250 \mathrm{~nm}$ thick PTO/STO film and bulk PTO (see Ref. 2). (c) Temperature evolution of the FWHM of (004) reflection and (d) the Pertsev phase diagram (see Ref. 12) of PTO. The misfit strains $\mathrm{S}_{\mathrm{m}}$ calculated for the PTO film between 70 and $550^{\circ} \mathrm{C}$ are reported in the diagram.

Figure 3 The temperature evolution of Raman spectra recorded on a film cross section in (a) a $\mathrm{VH}$ polarization configuration and (b) for the hard mode wavenumbers in a $250 \mathrm{~nm}$ thick PTO/STO film. Schematic representations of the VH polarization configuration and of Raman measurements on a film cross section are given in (c). 


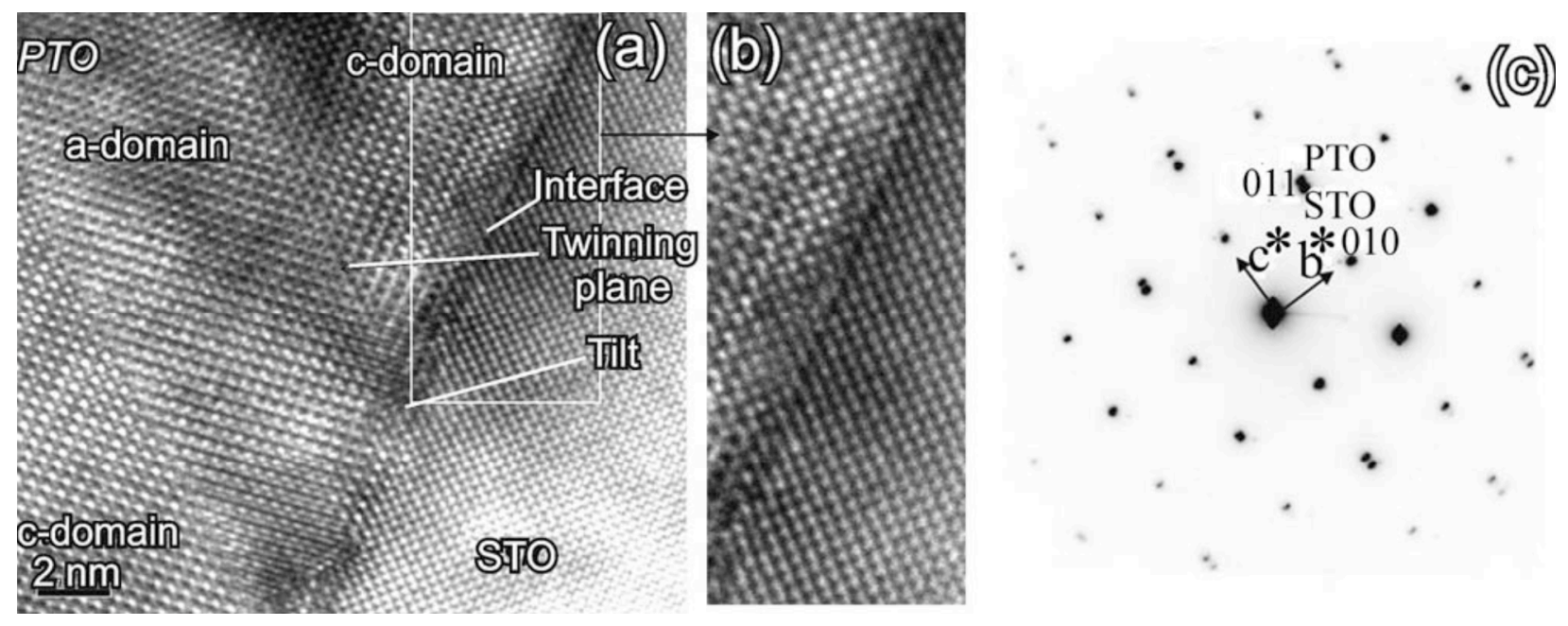

Fig. 1 

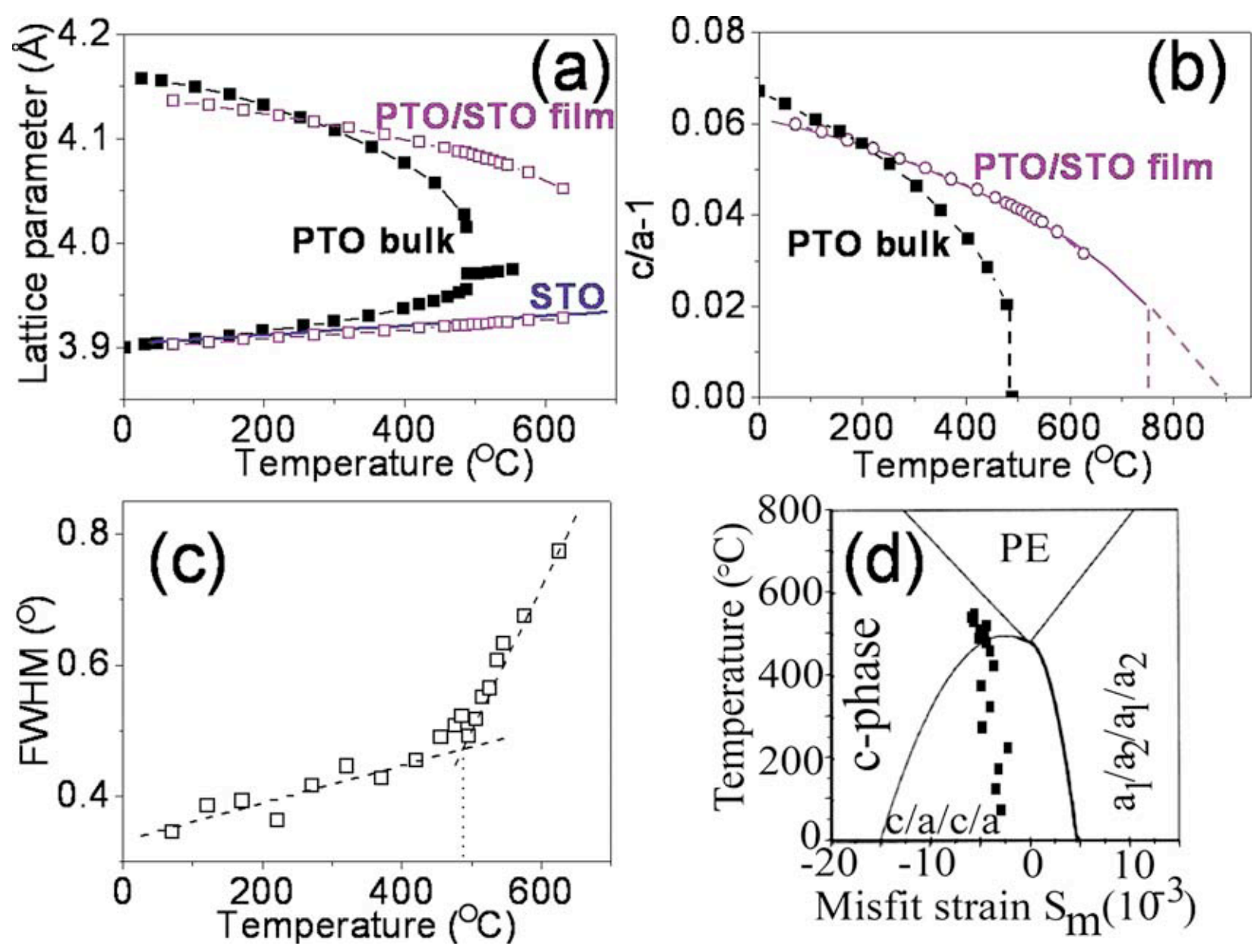

Fig. 2 

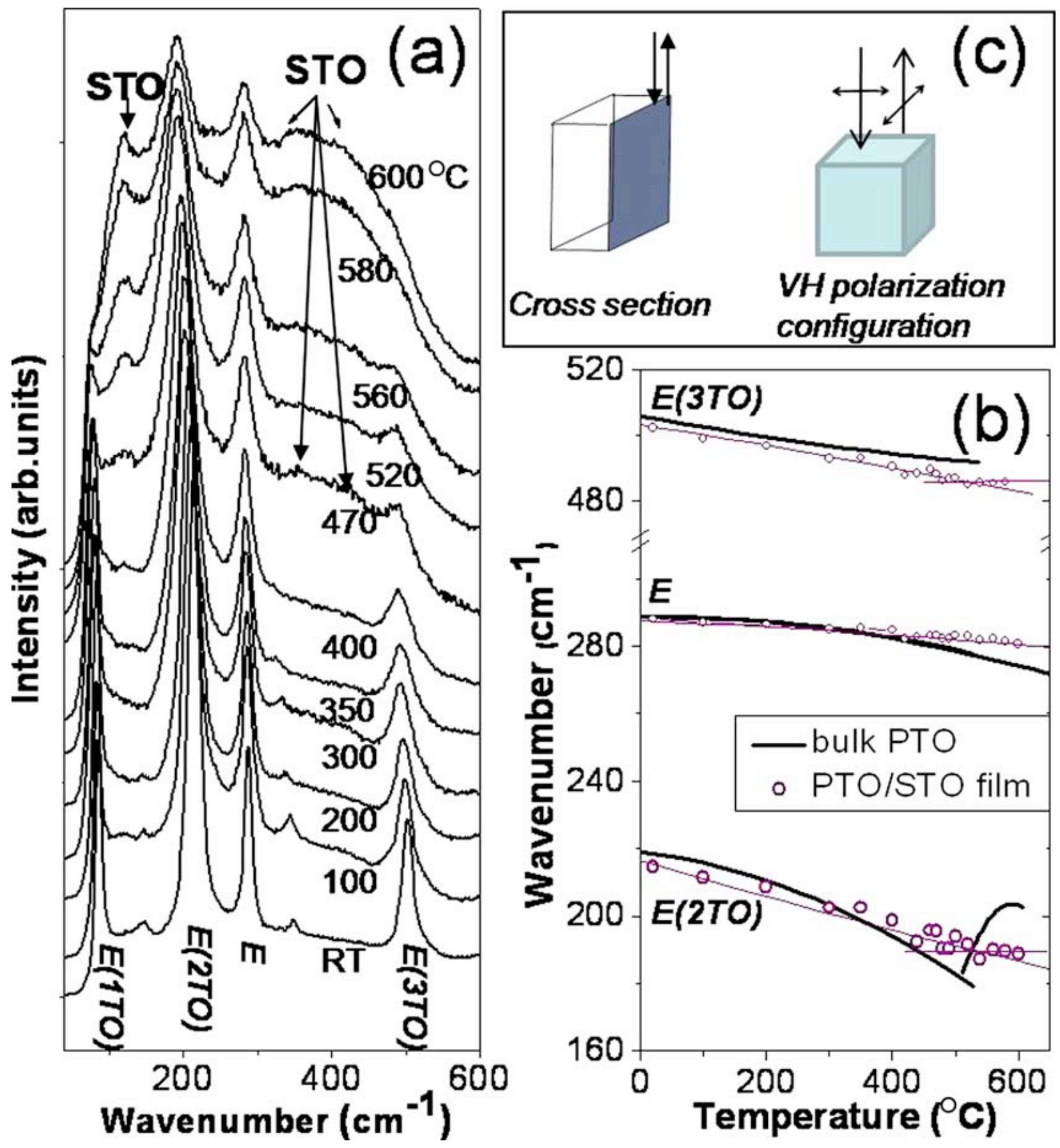

Fig. 3 